\title{
Efeito Casimir em meios dielétricos
}

\author{
Rocha, J.R; Orlando, M.T. D \\ 1 Programa de Pós-Graduação em Física, Universidade Federal do Espírito Santo, Vitória, ES, Brasil.
}

* e-mail: Jeffersonrocha2004@ig.com.br

\begin{abstract}
Resumo
Em 1948, Hendrik Casimir demonstrou que, duas placas paralelas condutoras e neutras sofrem uma força atrativa devido a flutuações do campo eletromagnético no vácuo. Neste trabalho, foi investigado o efeito Casimir entre dois semiespaços paralelos separados por uma distância d, e de constantes dielétricas $\varepsilon_{1}(\omega)$ e $\varepsilon_{2}(\omega)$ intercalados por um espaço de constante dielétrica $\varepsilon_{3}(\omega)$. Foram investigadas as forças entre as superfícies metálicas paralelas imersas entre os espaços e, discutida a contribuição dos plasmons no efeito Casimir nessas condições de contorno.
\end{abstract}

\begin{abstract}
In 1948, Hendrik Casimir showed that two conductive parallel and neutral plates experience an attractive force due to fluctuations of the electromagnetic field in a the vacuum state. In this study, was investigated the Casimir effect between two semi spaces separated by a distance d, and dielectric constants $\varepsilon_{1}(\omega)$ and $\varepsilon_{2}(\omega)$ interspersed by a space which dielectric constant is $\varepsilon_{3}(\omega)$. Were investigated the forces between the parallel metal surfaces immersed among the spaces and discussed the contribution of plasmons in the Casimir effect in these boundary conditions.
\end{abstract}

Keywords (Palavras chaves): Casimir, Dielectrics, plasmons.

\section{Introdução}

A teoria quântica para o campo eletromagnético na ausência de qualquer fonte foi formulada por Born, Heisemberg e Jordan em 1926. A primeira aplicação foi feita por Dirac em 1927 que, tratou da emissão e absorção de radiação. A eletrodinâmica quântica (QED) prediz a existência de flutuações no campo eletromagnético mesmo na ausência de fontes, isto é um campo eletromagnético de vácuo, relacionado à energia de ponto zero ou energia de vácuo $E_{0}=\frac{1}{2} \hbar \omega$. Em 1948, H.B.Casimir [1], demonstrou que uma das consequências da energia de ponto zero é uma força atrativa $F$ entre duas placas condutoras, paralelas e neutras separadas por uma distância d:

$$
F=-\hbar c \frac{\pi}{240} \cdot \frac{1}{d^{4}}
$$

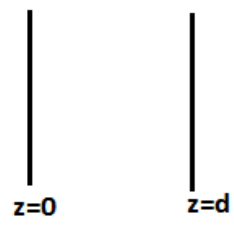

Figura 1: Placas condutoras separadas por d
A generalização da teoria de Casimir para placas condutoras (Figura 1) pode ser feita considerando se se o caso de um meio dielétrico cuja constante é $\varepsilon_{3}(\omega)$ entre dois semiespaços de constantes dielétricas $\varepsilon_{1}(\omega)$ e $\varepsilon_{2}(\omega)$ estes meios ocupam as regiões $0 \leq \mathrm{z} \leq \mathrm{d}, \mathrm{z}<0$ e $\mathrm{z}>\mathrm{d}$ respectivamente como mostrado na figura 2 .

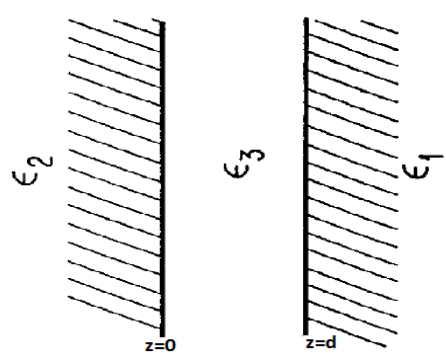

Figura 2: Placas semi-infinita separadas por uma distância d separadas por uma camada de constante dielétrica $\varepsilon_{3}$

O cálculo da força entre os dois espaços semi-infinitos é feito com base na energia de ponto zero do campo 
eletromagnético, $E=\sum_{n} \frac{1}{2} \hbar \omega_{n}$ onde $\omega_{n}$ são os modos de frequência na situação retratada na (figura 2) [2].

\section{Teoria}

\subsection{Quantização do campo eletromagnético e energia de ponto zero}

A quantização do campo eletromagnético implica em escrever os observáveis em termos de operadores que aumentam ou, diminuem o número de certas quantidades discretas no sistema, essas quantidades são conhecidas como quanta de excitação. Essa descrição do campo eletromagnético em termos de operadores pode ser feita reescrevendo o Hamiltoniano do campo em termos de quantidades fundamentais. Dessa forma, escrevendo a hamiltoniana do campo eletromagnético em $(2)[3,4,5]$ :

$$
\mathcal{H}=\int \frac{1}{2 \mu_{0}} B^{2}+\frac{\varepsilon_{0}}{2} E^{2} d^{3} x
$$

Reescrevendo (2) em termos do quadri-potencial $A^{\mu} \equiv$ $(\Phi, \vec{A})$ lembrando que:

$$
\begin{gathered}
\vec{E}=-\vec{\nabla} \Phi-\overrightarrow{A(r)} \\
\vec{B}=\vec{\nabla} \times \vec{A} \\
\mathcal{H}=\frac{1}{2} \int\left\{\frac{1}{\varepsilon_{0}} \Pi^{2}(r)+\varepsilon_{0} c^{2}[\vec{\nabla} \times \vec{A}]^{2}\right\}
\end{gathered}
$$

Onde $\Pi(r)$ é o momento canônico conjugado. Explicitamente, $\Pi(r)=-\varepsilon_{0} E(r) \quad$ e, assumindo a dependência espacial $\quad \boldsymbol{A}(\overrightarrow{\boldsymbol{r}})=\boldsymbol{A}(\mathbf{0}) e^{i k r} ., \quad$ o Hamiltoniano pode ser reescrito em termos do espaço dos momentos,

$$
\mathcal{H}=\int\left(\frac{1}{\varepsilon_{0}} \Pi(k)^{2}+\varepsilon_{0} k^{2} A(k)^{2}\right) d k^{3}
$$

A hamiltoniana (5) pode então ser reescrita em termos de operadores (6) e (7):

$$
\begin{aligned}
& a(k)=\sqrt{\frac{\varepsilon_{0}}{2 \hbar w}}\left[w \boldsymbol{A}(k)+\frac{i}{\varepsilon_{0}} \boldsymbol{\Pi}(k)\right] \\
& a^{*}(k)=\sqrt{\frac{\varepsilon_{0}}{2 \hbar w}}\left[w \boldsymbol{A}(k)-\frac{i}{\varepsilon_{0}} \boldsymbol{\Pi}(k)\right] \\
& \mathcal{H}=\sum \int \hbar w\left[a^{*}(k) a(k)+\frac{1}{2}\right] d k^{3}
\end{aligned}
$$

O Hamiltoniano em (8) é o idêntico ao oscilador harmônico:

$$
\left[\hat{\mathrm{A}}(k), \Pi\left(k^{\prime}\right)\right]=\mathrm{i} \hbar\left(k-k^{\prime}\right)
$$

$$
\left[\hat{\mathrm{a}}(k), \hat{\mathrm{a}}\left(k^{\prime}\right)\right]=i \hbar\left(k-k^{\prime}\right)
$$

Vale ressaltar que a quantização do campo eletromagnético proposta nessa seção foi feita no gauge de Coulomb isto é,

$$
\begin{gathered}
\Phi=\text { constante } \\
\vec{\nabla} \cdot \vec{A}=0
\end{gathered}
$$

Com base no hamiltoniano (9) é possível encontrar os autovalores de energia associados ao campo eletromagnético quantizado no estado fundamental. Aplicando o operador aniquilação no estado fundamental[ $[3,4,5]$ :

$$
\left.a_{k \lambda} 0\right\rangle=0
$$

A energia deste estado é a energia de ponto zero $E_{0}$ :

$$
E_{0}=\langle 0|H| 0\rangle
$$

$$
E_{0}=\sum_{k} \frac{\hbar w}{2}
$$

\subsection{O efeito Casimir}

O efeito CASIMIR [1] descreve a força atrativa entre duas placas perfeitamente condutoras separadas por uma distância d no vácuo (ver figura 1) esse efeito ocorre devido a flutuações do campo eletromagnético nesse estado. Considerando uma cavidade de dimensões $L_{x}$, $L_{y}$ e $L_{z}$, com as possíveis vibrações da cavidade isto é, $k_{x}=\frac{n}{L_{X}} \pi, k_{y}=\frac{l}{L_{Y}} \pi, k_{Z}=\frac{m}{L_{Z}} \cdot$, sendo $\mathrm{n}$,I e $\mathrm{m}$ números positivos inteiros.

$$
k=\sqrt{k_{x}^{2}+k_{y}^{2}+k_{z}^{2}}=\sqrt{k^{2}+k_{z}^{2}}
$$

Para cada número de onda $\mathrm{k}_{\mathrm{x}}$, $\mathrm{k}_{\mathrm{y}}$ e $\mathrm{k}_{\mathrm{z}}$ há dois modos de polarização, exceto para $n_{i}$ igual a zero, pois, nesse caso há apenas um modo de polarização. Para $k_{x}$ e ky esse fato não tem relevância uma vez que devido a $L$ grande, $k_{x}$ e $k_{y}$ podem ser tratadas como variáveis contínuas. Assim, igualando a energia de ponto zero à energia eletromagnética na cavidade para $L$ muito maior que Lz:

$$
\left.\sum \frac{2 \hbar w}{2}=\sum_{n, l, m} \pi \hbar c \sqrt{\left[\frac{n^{2} \pi^{2}}{L^{2}}+\frac{l^{2} \pi^{2}}{L}+\frac{m^{2} \pi^{2}}{L_{z}^{2}}\right]}\right)
$$

O fator 2 na equação (16) ocorre devido aos dois possíveis modos de polarização. Na situação física de interesse, $L z=d$, pois $L$ é muito maior que $L z$. Assim, a energia a uma distancia d da cavidade é:

Ou, escrito de outra forma: 


$$
E(d)=\frac{L^{2}}{\pi^{2}} \hbar c \sum_{m} \int_{0}^{\infty} d k_{x} \int_{0}^{\infty} d k_{y}\left(k_{x}^{2}+k_{y}^{2}+\frac{m^{2} \pi^{2}}{d^{2}}\right)
$$

A equação (17) leva a um valor infinito para a energia de ponto zero em um volume finito. Se d for feito arbitrariamente grande,

$$
E(\infty)=\frac{L^{2}}{\pi^{2}} \hbar c \frac{d}{\pi} \int_{0}^{\infty} d k_{x} \int_{0}^{\infty} d k_{y} \int_{0}^{\infty} d k_{z}\left(k_{x}^{2}+k_{y}^{2}+k_{z}^{2}\right)^{1 / 2}
$$

A equação (18) leva também a um valor infinito, portanto, a energia potencial $U$ (d) quando as placas estão separadas por uma distância d é dada pela diferença entre as equações (17) e (18). Essa diferença é infinita, contudo, é possível extrair um significado físico a partir de um valor finito. Transformando a diferença $U(d)=E(d)-E(\infty)$ para coordenadas polares $(r, \theta): \cdot$.

$$
\begin{gathered}
U(d)=\frac{L^{2} \hbar c}{\pi^{2}} \sum_{m} \int_{0}^{\pi / 2} d \theta \int_{0}^{\infty}\left(r^{2} \operatorname{sen}^{2} \theta+r^{2} \cos ^{2} \theta+\frac{m^{2} \pi^{2}}{d^{2}}\right)^{1 / 2} r d r \\
-\left(\frac{d}{\pi}\right) \int_{0}^{\infty} d k_{z} \int_{0}^{\infty} r\left(r^{2} \operatorname{sen}^{2} \theta+r^{2} \cos ^{2} \theta+k_{z}^{2}\right)^{1 / 2} d r \int_{0}^{\pi / 2} d \theta
\end{gathered}
$$

De maneira a resolver o problema da regularização, isto é a diferença entre duas grandezas infinitas, é introduzida uma função de corte no integrando:

$$
\begin{gathered}
U(d)=\frac{L^{2} \hbar c}{\pi^{2}} \frac{\pi}{2}\left[\sum_{m=0} \int_{0}^{\infty} r\left(r^{2}+\frac{m^{2} \pi^{2}}{d^{2}}\right)^{1 / 2} f\left(r^{2}+\frac{m^{2} \pi^{2}}{d^{2}}\right)^{1 / 2}\right]- \\
\frac{d}{\pi} \int_{0}^{\infty} r\left(r^{2}+k_{z}^{2}\right)^{1 / 2} d r d k_{z} f\left(\left(r^{2}+k_{z}^{2}\right)^{1 / 2}\right.
\end{gathered}
$$

Introduzindo agora a variável $t=\frac{d}{\pi} k_{z}$ e $u=\frac{d^{2}}{\pi^{2}} r^{2}$,

$$
\begin{gathered}
U(d)=\frac{L^{2} \hbar \pi^{2} c}{4 d^{3}}\left[\sum_{m=0}^{\infty} \int_{0}^{\infty} \sqrt{\left(m^{2}+u\right)} f\left(\frac{\pi}{d}\left[m^{2}+u\right]^{\frac{1}{2}} d u\right)-\right. \\
\int_{0}^{\infty} d t \int_{0}^{\infty}\left(u+t^{2}\right)^{1 / 2} f\left(\frac{\pi}{d}\left[u+t^{2}\right]^{1 / 2} d u\right]
\end{gathered}
$$

Definindo a função $f(t)$ como:

$$
f(t)=\int_{0}^{\infty}\left(u+t^{2}\right)^{1 / 2} f\left(\frac{\pi}{d}\left[u+t^{2}\right]^{1 / 2} d u\right.
$$

A diferença entre a série e a integral na equação (21) pode ser reescrita da seguinte forma:

$$
U(d)=\frac{\pi^{2} \hbar c}{4 d^{3}}\left[\frac{1}{2} F(0)+\sum_{m=0}^{\infty} f(m)-\int_{0}^{\infty} f(t) d t\right]
$$

Aplicando se a fórmula de Euller-maclaurin:

$$
\begin{aligned}
\sum_{m=0}^{\infty} f(m)-\int_{0}^{\infty} & f(t) d t=-\frac{1}{2}[f(0)+f(\infty)] \\
+ & \frac{1}{12}\left[f^{\prime}(\infty)-f^{\prime}(0)\right] \\
& +\frac{1}{720}\left[f^{\prime \prime \prime}(\infty)-f^{\prime \prime \prime}(0)\right]
\end{aligned}
$$

Aplicando a equação (24) em (23):

$$
U(d)=-\frac{\pi^{2} \hbar c}{720 d^{3}}
$$

A equação (25) é a energia associada a duas placas condutoras paralelas no vácuo, isto é a energia de Casimir. A força associada à energia descrita em (25) é encontrada calculando o gradiente da energia potencial,

$$
F(d)=-\frac{\pi^{2} \hbar c}{240 d^{4}} A
$$

O sinal negativo em (26) se deve ao fato de que a força nessa configuração de placas paralelas no vácuo é atrativa[3,4,5,8,10].

\subsection{0 efeito Casimir em meios dielétricos}

A extensão do efeito Casimir para meios dielétricos pode ser feita a partir da teoria de Lifishitz[7]. Especificamente, para a situação ilustrada na figura 2, em que as constantes dielétricas são respectivamente[1, 2,7,10,11]:

$$
\varepsilon(z)=\left\{\begin{array}{lc}
\varepsilon_{1} & z<0 \\
\varepsilon_{3} & 0<z<d \\
\varepsilon_{2} & z>d
\end{array}\right.
$$

As componentes diádicas de Green para as condições (27) devem ser expressas em termos dos modos TE (transversal elétrico ou $\mathrm{H}$ ) e TM (transversal magnético ou E), dados pelas funções escalares de Green:

$$
\begin{aligned}
& \left(-\frac{\partial^{2}}{\partial z^{2}}+k^{2}-w^{2} \varepsilon\right) g^{H}\left(z, z^{\prime}\right)=\delta\left(z-z^{\prime}\right) \\
& \left(-\frac{\partial}{\partial z} \frac{1}{\varepsilon} \frac{\partial}{\partial z^{\prime}}+\frac{k^{2}}{\varepsilon}-w^{2}\right) g^{E}\left(z, z^{\prime}\right)=\delta\left(z-z^{\prime}\right)
\end{aligned}
$$

de forma geral $\varepsilon=\varepsilon(z)$ e $\varepsilon^{\prime}=\varepsilon^{\prime}\left(z^{\prime}\right)$. As componentes não nulas de g são:

$$
\begin{aligned}
g_{x x} & =\frac{1}{\varepsilon} \delta\left(z-z^{\prime}\right)+\frac{1}{\varepsilon} \frac{\partial}{\partial z} \frac{1}{\varepsilon \prime} \frac{\partial}{\partial z^{\prime}} g^{E} \\
g_{y y} & =w^{2} g^{H} \\
g_{z z} & =-\frac{1}{\varepsilon} \delta\left(z-z^{\prime}\right)+\frac{k^{2}}{\varepsilon \varepsilon \prime} g^{E}
\end{aligned}
$$




$$
\begin{aligned}
& g_{x z}=i \frac{k}{\varepsilon \varepsilon^{\prime}} \frac{\partial}{\partial z} g^{E} \\
& g_{z x}-i \frac{k}{\varepsilon \varepsilon^{\prime}} \frac{\partial}{\partial z} g^{E}
\end{aligned}
$$

Tomando o limite $z=z^{\prime}$,

$g_{k k}=\left(w^{2} g^{H}+\frac{k^{2}}{\varepsilon \varepsilon^{\prime}} g^{E}+\frac{1}{\varepsilon \varepsilon^{\prime}} \frac{\partial}{\partial z^{\prime}} g^{E}\right) z=z^{\prime} \mid$

Quando a segunda interface (figura 2) é deslocada de uma quantidade $\delta d$,

$\delta \varepsilon(z)=\delta d .\left(\varepsilon_{2}-\varepsilon_{3}\right) \delta(z-d)$

Seja A área da seção transversa de uma da região imersa entre os meios dielétricos,

$$
\frac{\delta E}{A}=\frac{i}{2} \int \frac{d w}{2 \pi} \frac{d k}{(2 \pi)^{2}} \delta \varepsilon(z) g_{k k}(z, z, k, w)=-F \delta d
$$

Onde a força por unidade de área é:

$$
F=\frac{i}{2} \int \frac{d w}{2 \pi} \frac{d k}{(2 \pi)^{2}}\left(\varepsilon_{2}-\varepsilon_{3}\right) g_{k k}(d, d, k, w)
$$

Levando em conta que $\mathrm{g}^{\mathrm{H}}, \mathrm{g}^{\mathrm{E}}$ e $\frac{1}{\varepsilon} \frac{\partial}{\partial z} \frac{1}{\varepsilon} \frac{\partial}{\partial z^{\prime}}$ enquanto $\varepsilon \varepsilon^{\prime}$ é descontínua $z$ se aproxima de $z$ ' por lados opostos da interface então o termo $\frac{k^{2}}{\varepsilon \varepsilon^{2}} g^{E}$ é aproximadamente $\frac{k^{2}}{\varepsilon_{1} \varepsilon_{2}} g^{E}$ deve se avaliar apenas um lado da interface, assim , a função de Green $g^{H}$ para z,z'>d é:

$$
\left.g^{H}\left(z, z^{\prime}\right)=\frac{1}{2 k_{2}}\left(e^{-k_{2}}\left|z-z^{\prime}\right|\right)+r e^{-k_{2}\left(z+z^{\prime}-2 d\right)}\right)
$$

Onde o coeficiente de reflexão é:

$$
\begin{aligned}
& r=\frac{k_{2-} k_{3}}{k_{2+} k_{3}}+\frac{4 k_{2} k_{3}}{{k_{3}{ }^{2}-k_{2}{ }^{2}}^{2}} d^{-1} \\
& D=\frac{k_{2+} k_{3}}{k_{3-} k_{2}} \frac{k_{3+} k_{1}}{k_{3-} k_{1}} e^{2 k_{3} d}-1
\end{aligned}
$$

A função de Green $\mathrm{g}^{\mathrm{E}}$ tem a mesma forma da equação (39), apenas deve se levar em conta que k é substituído por $\equiv k^{\prime}$, avaliando a função de Green apenas fora da interface (Figura 2) encontra se a força por unidade de área[3, 4, 5, 8,10]:

$F=\frac{i}{2} \int \frac{d w}{2 \pi} \frac{(d k)}{(2 \pi)^{2}}\left\{\left[k_{3}-k_{2}+2 k_{3} D^{-1}\right]+\left[k_{3}-k_{2}+2 k_{3} D^{\prime-1}\right]\right\}$

$\mathrm{Na}$ equação (42), o primeiro colchete vem dos modos TE da função de Green e o segundo colchete da parte $\mathrm{TM}$, os primeiros termos em cada colchete que, não fazem referencia a distancia, podem ser vistos como a mudança na energia por unidade de volume do sistema. Estes termos representam a energia eletromagnética necessária para substituir o meio 2 pelo meio 3 o termo restante, que depende da distancia, é à força de Casimir['1]. Realizando uma rotação complexa na frequência:

$w \rightarrow i \varsigma$

$k^{2}=K^{2}+\varepsilon \zeta^{2}$
A partir da rotação proposta em (44),

$$
F=-\frac{1}{8 \pi^{2}} \int_{0}^{\infty} d \varsigma \int_{0}^{\infty} 2 k_{3}\left(D^{-1}+D^{-1 \prime}\right) d k^{2}
$$

Em particular, se o meio intermediário é o vácuo $\varepsilon_{3=} 1$ e $\varepsilon_{2}=\varepsilon_{1}=\infty$ e $k_{1}^{\prime}=k_{2}^{\prime}=0$ recupera se a força de Casimir para duas placas condutoras paralelas separadas por uma distancia d conforme expressão (26). De forma geral, se o meio intermediário não é o vácuo,

$$
\begin{gathered}
F=-\frac{1}{8 \pi^{2}} \int_{0}^{\infty} d \varsigma \int_{\zeta^{2} \varepsilon}^{\infty} \frac{4 k^{2}}{e^{2 k d}-1} d k^{2} \\
\boldsymbol{F}=-\frac{\boldsymbol{\pi}^{2}}{\mathbf{2 4 0 \sqrt { \varepsilon }}} \frac{\mathbf{1}}{\boldsymbol{d}^{4}}
\end{gathered}
$$

A equação (47) é a generalização do resultado encontrado em (26) imersa entre meios dielétricos. [7,10,11]

\subsection{Influência dos Plasmons no efeito Casimir}

Uma onda evanescente que percorre uma superfície com um comprimento de onda específico é chamada plasmon (ver figura 3). A energia de ponto zero a uma distância d da superfície é:

$E(d)=\sum_{n} \frac{\hbar w_{n a}}{2}+\sum_{n} \frac{\hbar w_{n b}}{2}$

Onde os índices (a) e (b) são os dois modos de vibração na superfície na equação (48)

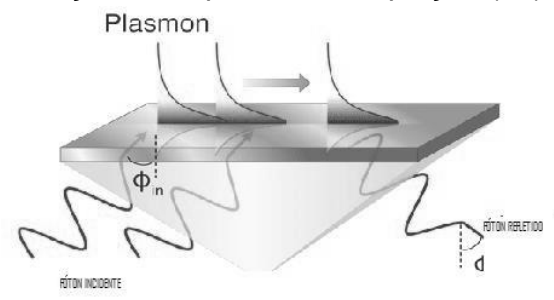

Figura 3: Plasmon se propagando em uma superfície metálica. Na figura o fóton incidente e refletido. Fonte: referencia [8]

$$
E(d)=\frac{\hbar L^{2}}{4 \pi} \int_{0}^{\infty}\left[\sum_{N} w_{N A}(k)+\sum_{N} w_{N B}(k)\right] k d k
$$

De acordo com Bordag. Et.al [9]:, a energia de ponto zero $E_{0}$, Para o dielétrico tem uma contribuição da energia do fóton incidente e dos plasmons de superfície[9]:

$E_{0}=E_{\text {Plasmon }}+E_{\text {Fóton }}$

\section{Resultados e discussão}

Foram discutidas a quantização do campo eletromagnético no gauge de Coulomb e o efeito da radiação no estado do vácuo sobre uma cavidade em forma de paralelepípedo cuja uma das dimensões é muito pequena comparada a área da secção 
transversal, isto é o efeito Casimir.com base no modelo de Llfishitz[7]:, foi feito o cálculo da energia de ponto zero para uma cavidade imersa em um meio dielétrico e encontrada a força a uma distancia d como foi feito para o vácuo $\mathrm{e}$, os resultados são convergentes com a teoria de Casimir como um caso limite conforme explicitado na secção 2.3,finalmente , verificamos os efeitos das vibrações de superfície (plasmons) sobre uma cavidade imersa entre meios dielétricos .através dessas análises, foram verificados os efeitos dos plasmons no efeito Casimir em dielétricos.

\section{Agradecimentos}

Os autores agradecem a CAPES pelo apoio financeiro.

\section{Referências}

[1] CASIMIR, H. B. G 1948 Proc. K. In: Ned. Akad. Wet. B. p. 793.

[2] VAN KAMPEN, N. G.; NIJBOER, B. R. A.; SCHRAM, K. On the macroscopic theory of van der Waals forces. Physics letters A, v. 26, n. 7, p. 307308, 1968.

[3]MILONNI, P. W. Casimir forces without the vacuum radiation field. Physical Review A, v. 25, n. 3, p. 1315, 1982.

. [4]LANDAU, Lev Davidovich et al. Electrodynamics of continuous media. Elsevier, 1984.

[5]GREINER, Walter. Quantum electrodynamics of strong fields. Springer Berlin Heidelberg, 1985.

[6]SCHWINGER, Julian. On gauge invariance and vacuum polarization. Physical Review, v. 82, n. 5, p. 664, 1951.

[7] LIFSHITZ, E. M. The theory of molecular attractive forces between solids. 1956.

[8 DIONNE, Jennifer A. Viewpoint: Mirror, Mirror.

Physics, v. 5, p. 38, 2012.

[9] BORDAG, Michael. The Casimir effect for thin plasma sheets and the role of the surface plasmons. Journal of Physics A: Mathematical and General, v. 39, n. 21, p. 6173, 2006.

[10] SCHWINGER, Julian; DERAAD, Lester L.; MILTON, Kimball A. Casimir effect in dielectrics. Annals of Physics, v. 115, n. 1, p. 1-23, 1978.
[11]CHEN, F. et al. Control of the Casimir force by the modification of dielectric properties with light. Physical review B, v. 76, n. 3, p. 035338, 2007. 
